\title{
Malnutrición fetal en el Hospital Regional de Chillán. Experiencia de un año: 1975-1976
}

En la actualidad se engloba a un grupo de recién nacidos de bajo peso de nacimiento con el nombre de Malnutridos Fetales (MF). En ellos

* Pediatras, Unidad de Recién Nacidos, Servicio de Pediatría, Hospital Regional de Chillán.

**Intemo de Medicina, Universidad de Concepción.

***Enfermeras Universitarias, Unidad de R. N., Servicio de Pediatría, Hospital Regional de Chillán.
Dres. Washington Melo*, Emilia Toro*, Carlos Castillo* Jaime Lama*, Virginia Valdés*; Sr. Victor Farfán ${ }^{* *}$ y Srtas. María Paz Soler*** y Dinely Ibáñez***.

existe durante el embarazo una deprivación de nutrientes tan prolongada como para repercutir seriamente en su crecimiento intrauterino, quedando ubicados en o bajo el percentil 10 de las tablas edad gestacional-peso de la Dra. Lubchenco. Este grupo debe distinguirse, entre otros, de los prematuros verdaderos. con los cuales pueden confundirse. 
La etiología de la MF está relacionada con antecedentes de toxemia, colestasia, gemelaridad, placenta previa, y anomalias placentarias y de cordón. Hay un 55 a $65 \%$ de casos en que no existe ningún antecedente patológico durante el embarazo, ni se encuentran anomalías placentarias o de cordón. No hay pruebas fehacientes que el estado nutritivo o las bajas condiciones socioeconómicas de la madre sean también factores etiológicos.

Es importante detectar a los MF porque presentan una mayor incidencia de ciertas patologías durante el embarazo, parto o período postnatal como sufrimiento fetal, asfixia neonatal, hipoglicemia, etcétera.

Estudios a largo plazo han comprobado que existe un compromiso en el desaxrollo pondoestatural, $\sin$ que se haya demostrado claramente alteración en otros parámetros.

\section{OBJETIVOS}

1. Se pretende evaluar el funcionamiento de nuestro Servicio de Recién Nacidos en base a una estadística puesta en marcha hace un año. Tomamos al MF como ejemplo de feto de alto riesgo para dicho propósito. Nuestro hospital, al no contar con los medios óptimos de laboratorio, instrumentales $y$ ambientales, refleja la realidad de hospitales de provineia, los cuales podrían aprovechar nuestra experiencia.

2. Queremos llevar la voz de alerta al equipo obstétrico respecto de la importancia en la precoz pesquisa de esta patología; ello debe lograrse estudiando más acuciosamente algunas enfermedades de embarazo como la toxemia, colestasia, placenta previa y retardo del crecimiento intrauterino. Buscamos obtener un producto en las mejores condiciones mediante conductas obstétricas definidas frente a patologías del embarazo y parto.

3. Por su parte el pediatra deberá mantener una conducta alerta y agresiva frente a este tipo de niños para detectar patologías propias de su condición, observarlos cuidosamente y tratarlos, ya que por tener muchos de ellos un peso mayor de $2.000 \mathrm{grs}$ se consideran erróneamente que están aptos para ser dados de alta junto a su madre.

\section{MATERIAL Y METODO}

Se revisarore los ingresos a las Unidades de Recién Nacidos Patológicos en el período de un año, comprendido entre el 1 de septiembre de 1975 y el 31 de agosto de 1976. En un total de 757 recién nacidos hospitalizados en dicho periodo, se encontraron 67 malnutridos fetales y 690 R.N. que no lo eran y que fueron hospitalizados por otras patologías; de estos 690 R.N., 538 eran de término adecuado a la edad gestacional, y 152 eran de pretérmino adecuado a la edad gestacional (tabla 1).

Tabla 1

DISTRIBUCION DE 757 R.N. HOSPITAL DE CHILLAN

\begin{tabular}{lc}
\hline Condición & .10 \\
\hline R.N. Término & 538 \\
R.N. Pretérmino & 152 \\
Malnutrido Fetal & 67 \\
\hline
\end{tabular}

Se consideraron malnutridos fetales a todos aquellos recién nacidos con retraso, de crecimiento intrauterino, ubicados en o bajo el percentil 10 de la gráfica edad gestacionalpeso de la Dra. Lubchenco, y en los cuales ese retraso se atribuyó a insuficiente nutrición intrauterina; por lo tanto se descartaron los escasos RN con malformaciones, infecciones cogestacionales y alteraciones genéticas. La edad gestacional se estimó de acuerdo a la fecha de última regla matema y al examen físico del recién nacido.

Respecto a las madres de los MF se revisaron sus antecedentes de control de embarazo, procedencia, paridad, edad, tipo de parto y se comparó su patología gestacional con la de las madres de los recién nacidos de término y pretérmino adecuados a la edad gestacional.

En los MF se estudió promedio de peso, compromiso de la talla, promedio de días de estada, su mortalidad, y se comparó la patología del parto y del período de hospitalización en las Unidades de RN con la patología de los recién nacidos de pretérmino y término adecuados a la edad gestacional.

\section{RESULTADOS Y COMENTARIOS}

En el período estudiado hubo 4.187 RN vivos, de los cuales 101 corresponden a malnutridos 
fetales con el 2,4\%; 67 de éstos fueron hospitalizados 10 , que corresponde al material de nuestra revisión.

En el grupo de MF encontramos $58,2 \%$ de multiparas, $11,9 \%$ de grandes multiparas y $29,8 \%$ de primiparas; de éstas, el $20 \%$ eran primiparas añosas y un $15 \%$ primíparas precoces (véase gráfico).

\section{GRAFICO № 1}

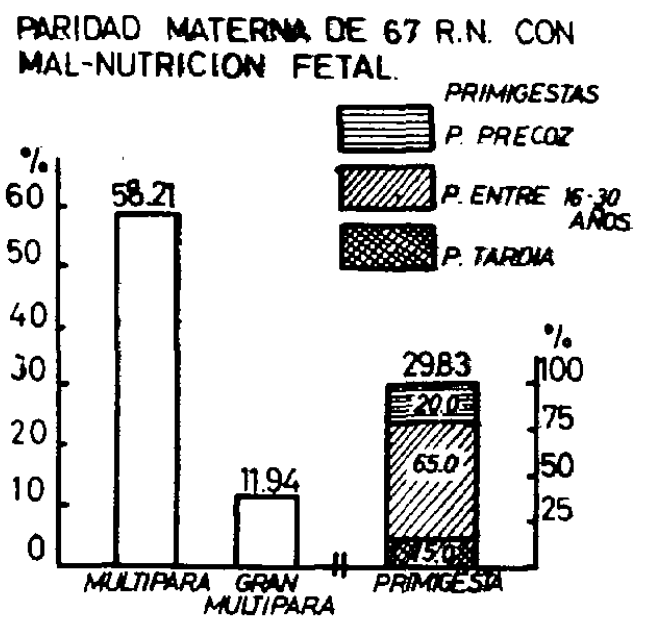

Estudiamos la procedencia de las madres de los MF y encontramos que $66,5 \%$ proviene de medios urbanos, especialmente de la ciudad de Chillán, y el $34,3 \%$ proviene de zonas rurales; esto lo relacionamos con el porcentaje de control de embarazos y encontramos que el $81,8 \%$ de las madres de medios urbanos controlaron su embarazo y en cambio sólo se controlaron el $34,7 \%$ de las madres de medios rurales (véase gráfico).

Estos datos revelan la situación del control obstétrico en Chillán, indicándonos la fatta de captación de las madres de medios rurales, a lo cual hay que añadir que aún existe un elevado porcentaje de partos en domicilio.

Analizando la patología del embarazo encontramos que el $10,4 \%$ de las madres de los MF presentaron toxemia, contra un $2,9 \%$ en los RNT AEG y $1,31 \%$ en los pretérmino AEG, señalándonos que la toxemia es un factor etiológico de malnutrición fetal.

El 3\% de madres de MF tenía antecedentes de colestasia gravídica; en cambio en los RNT AEG dicho porcentaje era de $0,37 \%$ y en los RN pretérmino AEG era de $0,65 \%$, siendo esto
GRAFICO $N^{\circ} 2$

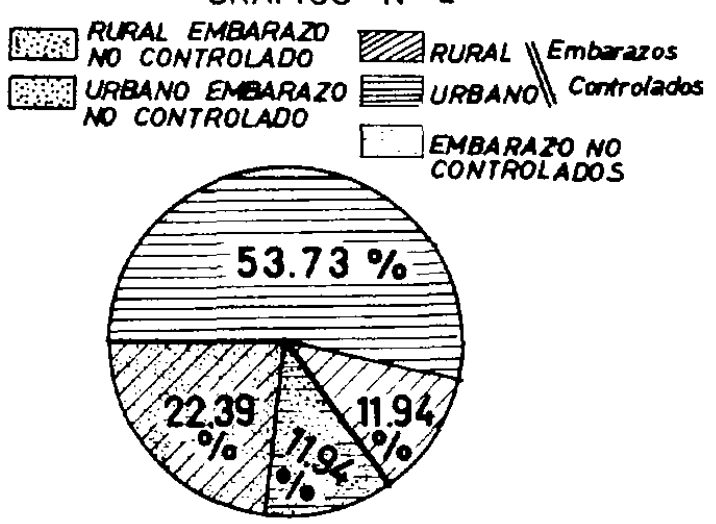

CONTROL DE EMBARAZO EN RELACION A LA PROCEDENCIA DE LA MADRE.

otro factor condicionante de malnestrición fetal. Tenemos la impresión que esta patología es poco frecuente en nuestra área.

La placenta previa estuvo presente en el $3 \%$ de las madres de MF contra un $0,55 \%$ en los RNT AEG y $0,65 \%$ èn los RN pretérmino AEG.

El $83,4 \%$ de los MF no presentaba antecedentes patológicos de embarazo, en comparación con un $90,1 \%$ de los RNT AEG y $97,5 \%$ de los RN pretérmino AEG. Este porcentaje en cuanto a los MF está mucho más alto que lo descrito en la literatura (55 a $65 \%$ ), lo cual nos indicaría una mala pesquisa de patologías del embarazo, placenta y cordón. (véase gráfico y tabla 3).

\begin{tabular}{|c|c|c|}
\hline \multicolumn{3}{|c|}{$\begin{array}{l}\text { GRAFICO No } 3 \\
\text { ENTES PATOLOGICO } \\
O \text { DE LAS MADRE }\end{array}$} \\
\hline $\begin{array}{l}\text { ANTECEDENTE } \\
\text { OBSTETRICO }\end{array}$ & CASOS & $\begin{array}{c}\text { PORCENTAJE } \\
5 \\
10\end{array}$ \\
\hline PRE-ECLAMPSIA & 7 & $-1045 \%$ \\
\hline COLOSTASIA & 2 & 2.99 \\
\hline GEMELARIDAO & 5 & -7.46 \\
\hline PLACENTA PREVIA & 2 & 2.99 \\
\hline SIN ANTECEDENTES & 53 & - \\
\hline
\end{tabular}

En los partos de los RN malnutridos fetales encontramos un $58 \%$ de partos normales, $28,3 \%$ de cesáreas las cuales se realizaron por 
Tabla 3

\begin{tabular}{|c|c|c|c|}
\hline \multicolumn{2}{|c|}{ Incudencia de toxemia en 757 R.N. Hosp. Chillán } & \multicolumn{2}{|c|}{ Incidencia de colostasia en 757 R.A. Hosp. Chillán } \\
\hline RN tér. & $2.9 \%$ & RNíter. & $0,37 \%$ \\
\hline RN Pr. & $1,3 \%$ & RN pr. & $0,65 \%$ \\
\hline MNF & $10,4 \%$ & MNF & $2,98 \%$ \\
\hline \multicolumn{2}{|c|}{ Incidencia de placenta previa en $757 \mathrm{RN}$} & \multicolumn{2}{|c|}{ Sin anteced patolog. embarazn en $757 \mathrm{R} . \mathrm{I}$} \\
\hline RN tér. & $0,55 \%$ & RN tér. & $96,1 \%$ \\
\hline RN pr. & $0,65 \%$ & RN pr. & $97,5 \%$ \\
\hline $\mathrm{MNF}$ & $2,90 \%$ & MNF & $83,4 \%$ \\
\hline
\end{tabular}

sufrimiento fetal agudo, rotura prematura de membranas, placenta previa, cesárea anterior; $8,9 \%$ nacieron por podálica, un $4,4 \%$ por fórceps. Hubo $7,46 \%$ de embarazos gemelares. En la Maternidad de Chillán se tiene un $73,9 \%$ de partos normales, un $21,2 \%$ de césareas; un $5,4 \%$ de fórceps y un $0,5 \%$ de podálicas. Llama la atención el mayor porcentaje de cesáreas. las cuales fueron indicadas por causas ajenas a la MF. Debe hacerse notar que en ningún caso se sospechó previamente la mala condición fetal que estudiamos en este trabajo, y la gran mayoría de los embarazos llegaron a término, agravando las condiciones de los RN.

En los RN analizados no hubo diferencias significativas en cuanto al sexo con un $52 \%$ de varones y un $48 \%$ de mujeres. (véase gráfico).

El promedio de peso de los malnutridos fetales de término fue de $2.232 \mathrm{grs}$, y en los de pretérmino fue de 1.810 grs; la mayoría de éstos se agruparon en las 35 a 36 semanas de gestación.

\section{GRAFICO No 5}

\section{DISTRIBUCION POR SEXO DE 67 R.N. CON M.N.F.}

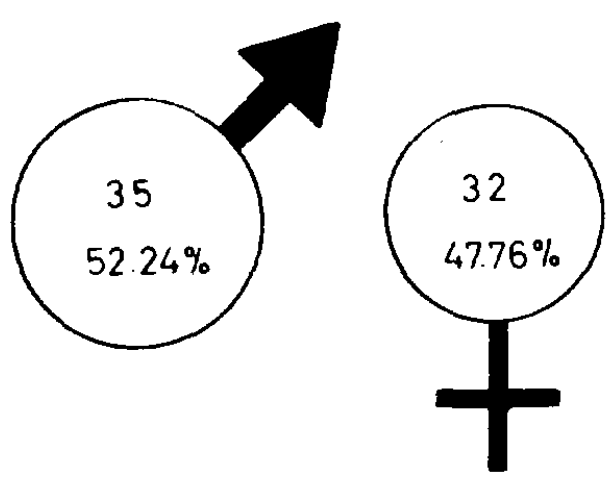

En un $48 \%$ hubo compromiso de talla según las tablas edad gestacional-talla de la Dra. Lubchenco, quedando bajo el percentil 10 , y $31,8 \%$ quedó bajo el percentil 25. (véase grafico).

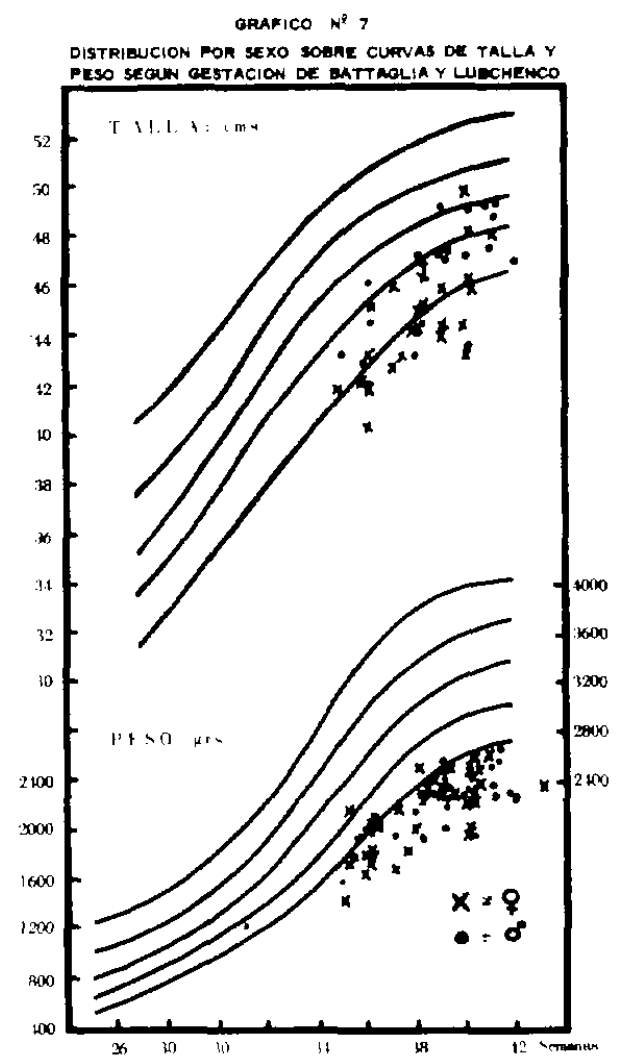

El $31 \%$ de los MF presentaron sufrimiento fetal agudo $y / 0$ crónico, contra un $17,8 \%$ en los RNT AEG y un $11.1 \%$ en los RN pretérmino AEG, lo que nos demuestra la importancia del sufrimiento fetal entre las causas de MF.

Un $2,9 \%$ de los $M F$ presentó asfixia neona- 
tal grave, en tanto esa patología se presentó en $7,06 \%$ de los RNT AEG y en $6,5 \%$ de los RN pretérmino AEG. Llama la atención el alto porcentaje de asfixia en los RNT y pretermino AEG, pero esto se explica, pues son RN hospitalizados por esta causa entre otras. En cuanto a asfixia moderada, ella estuvo presente en $14,9 \%$ de los $\mathrm{MF}$, en $8,17 \%$ de los RNT AEG y en $5,2 \%$ de los pretérmino AEG.

Tomando estos datos desde otro punto de vista, tenemos que el $40 \%$ de los $\mathrm{MF}$, el $33,1 \%$ de los RNT AEG y el $22 \%$ de los pretérmino AEG presentaron hipoxia perinatal, considerando por tales los que tuvieron sufrimiento fetal $y / o$ asfixia neonatal, ya que fueron comparados con recién nacidos patológicos. (véase gráfico y tabla 5).

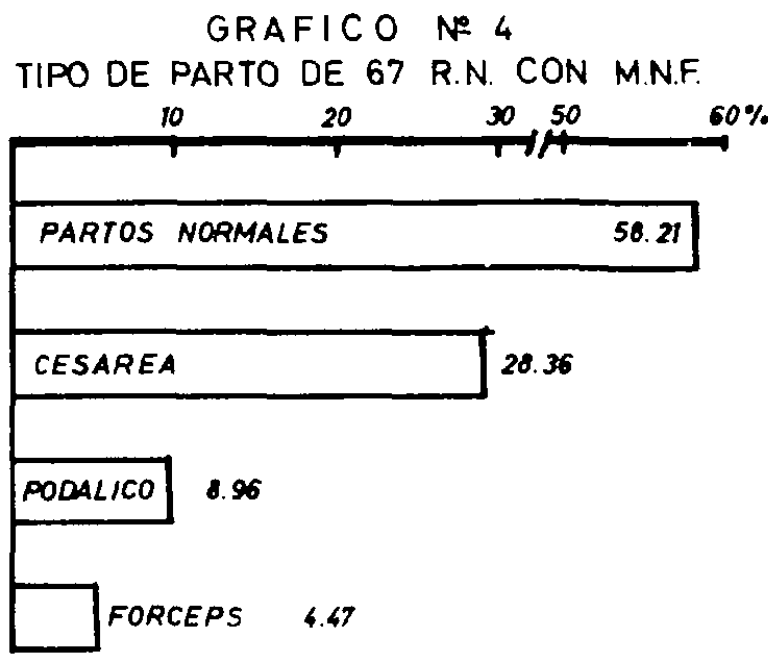

Tabla 5

PATOLOGIA DE PRE Y POSTPARTO IDE 67 RN CON MNF

\begin{tabular}{lrr}
\hline Patologia & Cases & $\%$ \\
\hline Sufrimiento fetal agudo y crónico & 21 & 31,34 \\
Asfixias graves & 2 & 2,99 \\
Asfixias moderadas & 10 & 14,92 \\
Sin asfixias & 40 & 59,72 \\
\hline
\end{tabular}

El $61 \%$ de los MF fue enviado de la sala de partos por presentar una patología agregada, y no por sus características de MF; el $30 \%$ fue enviado desde puerperio con posterioridad al examen pediátrico; el $19 \%$ volvió de su domicilio por infecciones intercurrentes (véase tabla 4).
Tabla 4

PROCEDENCIA IJE 67 RN. CON MNF

\begin{tabular}{llrr}
\hline Sala Puerperio & RN. tér. 18 & $90,0 \%$ & $26,86 \%$ \\
& RN. pret. 2 & $10,0 \%$ & $2,99 \%$ \\
& Total RN 20 & $100,0 \%$ & $29,85 \%$ \\
Sala Partos & RN. tér. 25 & $61,0 \%$ & $37,31 \%$ \\
& RN. pret. 16 & $39,0 \%$ & $23,88 \%$ \\
& Total RN 41 & $100,0 \%$ & $61,19 \%$ \\
Domicilio & RN. tér. 6 & $100,0 \%$ & $18,96 \%$ \\
\hline
\end{tabular}

El primer examen pediátrico de los malnutridos fetales fue realizado en un $61 \%$ en las primeras 12 horas de vida; entre las 12 y las 24 horas de vida se examinaron el $23,4 \%$ y un $16 \%$ con 24 o más horas (véase gráfico).

GRAFICO NN 6

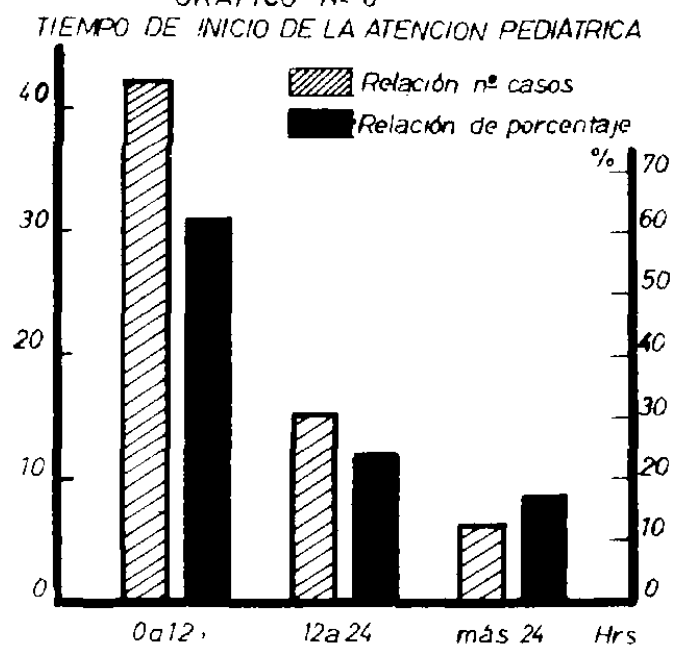

En cuanto a la patología postnatal encontramos:

1. Hipoglicemia en los $\mathrm{MF}$, en un $45 \%$; hipoglicemia en los RNT AEG, en un $0,55 \%$; hipoglicemia en los RN pretérmino AEG, en un 7,8\%.

Estas cifras nos dan a entender que los MF son más susceptibles a desarrollar hipoglicemia, ya que todos están sometidos a las mismas condiciones ambientales desfavorables (frío). Corrobora este hecho que el $55 \%$ de los MF enviados desde puerperio presenta hipoglicemia la que se hizo más evidente por la falta de temperatura adecuada en las salas de Obstetricia.

El $30 \%$ de las hipoglicemias de los MF necesitó el uso de corticoides para su tratamiento, lo que nos hablaría de una mala respuesta al tratamiento habitual estipulado en las normas de recién nacidos del SNS.

2. Estudiamos las hiperbilirrubinemias que no 
obedecen a incompatibilidad de grupo ni $\mathrm{Rh}$; encontramos un $34,3 \%$ en los $\mathrm{MF}$, un $28,6 \%$ en los RNT AEG y un $22,3 \%$ en los RN pretérmino AEG. El porcentaje en los malnutridos fetales puede explicarse por los antecedentes de hipoxia perinatal, poliglobulia, infecciones, hipoglicemia, factores todos que favorecen su producción. En los RNT AEG llama la atención el alto porcentaje de hiperbilirrubinemia, lo que se interpretó en parte por los antecedentes de asfixia neonatal y además por el crecimiento, uso de ocitósicos durante el parto. El porcentaje de hiperbilirrubinemia en los RN pretérmino AEG se explica por la inmadurez hepática propia de este grupo, agregándose las causas anteriormente dichas.

3. El $10 \%$ de los MF presentó poliglobulia; en cambio esta patología la encontramos sólo en un 1,1 y en $1,3 \%$, respectivamente de $\operatorname{los} \mathrm{RN}$ de término y pretérmino AEG. El alto porcentaje encontrado en MF se explica por hipoxia crónica (véanse tabias 6 y 7 ).

4. Ln $26,8 \%$ de los MF presentó infecciones intercurrentes.

5. Hubo un $5 \%$ de mortalidad en los MF contra un $3,8 \%$ en los RNT AEG y un $33,4 \%$ en los $\mathrm{RN}$ pretérmino AEG.

\section{Tabla 0}

\section{PATOLOGIA DE 67 RN CON MALNLTRIGION} FETAI.

\begin{tabular}{lcr}
\hline Patología & Caso. & \multicolumn{1}{c}{$\%$} \\
\hline Hipoglicemias & 30 & 44,78 \\
tratadas con Corticod. & & 30,0 \\
Infecciones & 18 & 26,86 \\
Hiperbilirrubinemia & 23 & 34,33 \\
Polighobulia & 7 & 10,45 \\
\hline
\end{tabular}

Tabla 7

\begin{tabular}{|c|c|c|c|}
\hline \multicolumn{2}{|c|}{$\begin{array}{l}\text { Inctdencta de hipoglic. } \\
\text { En } 757 \mathrm{RN} \text {, Hosp. Chillán }\end{array}$} & \multicolumn{2}{|c|}{$\begin{array}{l}\text { Incedenctade hiperblimul. } \\
\text { en } 757 R N \text {, Hosp. Chillán }\end{array}$} \\
\hline RNT & $0,55 \%$ & $\mathrm{RN} \mathrm{I}$ & $28.6 \%$ \\
\hline R N P & $7,80 \%$ & RN P & $22.3 \%$ \\
\hline $\mathrm{MNF}$ & $44,65 \%$ & MNF & $34.3 \%$ \\
\hline \multicolumn{4}{|c|}{$\begin{array}{c}\text { Inculencia de poliglobulia en } 757 \\
\text { RN del Hospital de Chillón }\end{array}$} \\
\hline & $\begin{array}{l}\text { RNitér. } \\
\text { RN Pret. } \\
\text { MNF }\end{array}$ & & \\
\hline
\end{tabular}

\section{CONCLUSIONES}

Podemos deducir lo siguiente:

- Necesidad imperiosa de captar a las embarazadas de rural para su control y atención profesional.

- Existen patologías del embarazo productoras de malnutrición fetal, como la toxemia, colestasia y placenta previa.

- Los MF tienen mayor incidencia de hipoxia perinatal que los RNT y los RN pret. AEG.

- Hay un porcentaje mayor de hipoglicemias en los MF, que no se observa en otros grupos sometidos a similares condiciones ambientales.

- La poliglobulia fue otra patología de relevante importancia en los MF frente a la cual hay que estar atento y realizar un tratamiento oportuno.

- Consideramos que las estadísticas del Servicio de Recién Nacidos han cumplido con su función y nos dan una clara visión del problema en nuestra localidad.

Nuestras metas próximas serán: conseguir la apertura de una Unidad de atención inmediata y observación del RN, de un policlínico de alto riesgo obstétrico; el conseguir entrenamiento de personal de Obstetricia en el buen manejo del recién nacidn; obtener normas obstétricas bien definidas de diagnóstico y tratamiento frente a patologías del embarazo y parto.

\section{RESUMEN}

Se revisaron los ingresos a las unidades de $\mathrm{RN}$ patológicos desde el 1 de septiembre de 1975 al 31 de agosto de 1976

Hubo 757 ingresos que se desglosan de la siguiente manera: $67 \mathrm{MF}, 538 \mathrm{RNT}, 152 \mathrm{RN}$ pretémino.

Se estudió la patología del embarazo, parto y período postnatal. Encontramos que el $81,8 \%$ de las madres de medios urbanos controló su embarazo, en cambió sólo el $34.3 \%$ de las-madres de medios rurales lo hizo.

En cuanto a las patologías de embarazo encontramos que el $10,4 \%$ de los MF tenía antecedentes de toxemia contra un $2.9 \%$ de los RTN y el $1.31 \%$ de los RN Pr. E1 $3 \%$ de los $\mathrm{MF}$ tenia antecedentes de colestasia; en cambio en los RNT un $0,37 \%$ y $0,65 \%$ en los RN Pr. El 3\% de los MF presentó pliacenta previa contra $0,55 \%$ en los RNI y $0.65 \%$ en los RN pretérmino.

El $40 \%$ de los MF presentó hipoxia perinatal. en los R.VI $33,1 \%$ y en los RN Pr. $22 \%$. 
Las patologías del período postnatal las constituyeron principalmente la hipoglicemia con $45 \%$ en los MF, sólo 0,55\% en los RNT AEG y $7,8 \%$ en los RN Pr. AEG. La poliglobulia en los MF fue de 10,4\%; en cambio sólo hubo $1,1 \%$ en los RNT y $1,31 \%$ en los RN Pr.

Tuvimos $5 \%$ de mortalidad en los MF, 3,8\% en los RNT y $33,4 \%$ en los RN Pr.

\section{REFERENCIAS}

Aluarado, S.; Valenzuela, J.; Schlager, G.; Ferreiro, M. y Rizzardini, M. Rev. Clin. Pediat. 43:9, 1972

Beargie, R. y James, V. Clín. Pediátr. N.A. Febrero 159. 1970.

Bravo, M.; Valenzuela, J. y RLzzardin, M. Pediatría 16, 173.

Cassady, G. Pediat. Clin. N.A. 17:79, 1970.

Drillien, C. Pediat. Clin. N.A. 17:9, 1970.

Falkner, F. Pediat. Clin. N.A. 17:5, 1970.

Gellis. Year Book de Pediatria 1975. Editorial Médica Panamericana, Buenos Aires, República Argentina, 1975.

Gwovich, L. y Carcur, E. Rev. Clín. Pediát. 41:1015, 1970

Grvouch, L. y León, M. Rev. Abril Pediat. 41:11, 1970.

Kontras, S. Pediat. Clin. N.K. 19:919, 1972.

Klaus, $M$. y Fanaroff, $A$. Aststencia del recién nacido de alto riesgo. Editorial Médica Panamericana, Buenos Aires, República Argentina, 1975.
Las Heras, J.; Dazzarola, P. y Rizzardini, M. Rev. Abril Pediat. 42:255, 1971.

Lubchenco, L. Clín. Pediat. N.A. 17:125, 1970.

Llanos, Neonatología 3:25, 1975.

Llanos, A. y Segovia, $\boldsymbol{N}$. Neonatología 3:29, 1975.

Llanrss, A.; Zegers, F. y Conalán, S. Retraso del crecimiento intrauterino. Grabación del Seminario de Actualización Perinatal. Hosp. Josefina Martínez de F., Puente Alto, Nov. 1975.

Meneghello, J. Pediatría, tomo I. Editorial Intermédica, Buenos Aires, República Argentina, 1972.

Mesa Redonda. Rev. Chil. Pediat. 39:117, 1968.

Oski, F. and Naiman, J. Philadelphia, 1972. W.B. Saunders.

Rzzzardint, M. Rev. Clin. Pediat. 44:281, 1973.

Rizzardin, M. y Dazzarola, P. Pediatría (Santiago) 12:97, 1969.

Rizzardint, M.; Dazzarola, P. y Las Heras, J. Pediatría 15: 357,1972

Servicuo Nacuonal de Salud de Chule. Normas para la atención del recién nacido. Edit. Gabriela Mistral, Santiago, 1973.

Silva, E. El hijo de la madre diabética, toxemia y con cobestasia intrahepática del embarazo. Grabación del curso de "Actualización en problemas de Perinatologia", Hosp. Roberto del Río, Santiago, julio, 1976.

Sinclair, J. Pediat. Clin. N.A. 17:147, 1970.

Usher, R. Pediat. Clin. N.A. 17:169, 1970.

Winick, M. Clín. Pediat. N.A. 17:69, 1970. 